\title{
A tomografia computadorizada multislice é ferramenta importante para o estadiamento e seguimento do câncer de mama?*
}

Is multislice computed tomography an important tool for breast cancer staging and follow-up?

\author{
Christiana Maia Nobre Rocha de Miranda ${ }^{1}$, Carla Jotta Justo dos Santos ${ }^{2}$, Carol Pontes de Miranda \\ Maranhão², Lucas de Pádua Gomes de Farias ${ }^{3}$, Igor Gomes Padilha ${ }^{3}$, Anna Carolina Mendonça \\ de Andrade ${ }^{4}$, Mayara Stephanie de Araujo Jatobá ${ }^{4}$
}

\begin{abstract}
Resumo O câncer de mama representa o tipo de câncer mais comum em mulheres e constitui a primeira causa de morte por câncer nesta população. As alterações extramamárias relacionadas ao câncer de mama desempenham papel relevante no prognóstico e tratamento desta entidade, sendo fundamental a realização do diagnóstico correto e das diversas alterações. A maioria dessas manifestações é proveniente do tratamento adotado ou de suas complicações e pode estar associada a comprometimento linfonodal, recorrência locorregional ou metástases a distância.

Unitermos: Tomografia computadorizada multislice; Estadiamento; Câncer de mama; Alterações extramamárias.
\end{abstract}

\begin{abstract}
Breast cancer is the most common cancer in women and the leading cause of cancer death among the female population. Extramammary findings related to breast cancer play an important role in the prognosis and treatment of such entity and the correct diagnosis of such alterations is essential for increasing the chance of cure. Most of the times, such manifestations result from complications arising from treatment, and may be associated with lymph node involvement, locoregional recurrence or distant metastasis.
\end{abstract}

Keywords: Multislice computed tomography; Staging; Breast cancer; Extramammary changes.

Miranda CMNR, Santos CJJ, Maranhão CPM, Farias LPG, Padilha IG, Andrade ACM, Jatobá MSA. A tomografia computadorizada multislice é ferramenta importante para o estadiamento e seguimento do câncer de mama? Radiol Bras. 2012 Mar/Abr;45(2):105-112.

\section{INTRODUÇÃO}

O câncer de mama é o segundo tipo de câncer mais frequente, sendo o mais prevalente entre as mulheres. Anualmente, cerca de $22 \%$ dos casos novos de câncer em mulheres são de mama ${ }^{(1)}$. Apesar da alta incidência, a mortalidade por câncer de mama vem decrescendo, graças à detecção

\footnotetext{
* Trabalho realizado na Clínica de Medicina Nuclear e Radiologia de Maceió (MedRadiUS), Maceió, AL, Brasil.

1. Doutora, Coordenadora do Setor de Tomografia Computadorizada da Clínica de Medicina Nuclear e Radiologia de Maceió (MedRadiUS), Professora de Radiologia e Diagnóstico por Imagem da Universidade Federal de Alagoas (UFAL), Maceió, AL, Brasil.

2. Membros Titulares do Colégio Brasileiro de Radiologia e Diagnóstico por Imagem (CBR), Médicas da Clínica de Medicina Nuclear e Radiologia de Maceió (MedRadiUS), Maceió, AL, Brasil.

3. Acadêmicos de Medicina, Monitores da Disciplina de Radiologia e Diagnóstico por Imagem da Faculdade de Medicina da Universidade Federal de Alagoas (UFAL), Maceió, AL, Brasil.

4. Acadêmicas da Faculdade de Medicina da Universidade Federal de Alagoas, Maceió, AL, Brasil.

Endereço para correspondência: Dra. Christiana Maia Nobre Rocha de Miranda. Rua Hugo Corrêa Paes, 104, Farol. Maceió, AL, Brasil, 57050-730. E-mail: maiachristiana@globo.com

Recebido para publicação em 23/10/2011. Aceito, após revisão, em 13/12/2011.
}

precoce e aos recursos tecnológicos de imagem disponíveis ${ }^{(\mathbf{2})}$.

Em razão da sua reduzida sensibilidade e especificidade, a tomografia computadorizada multislice (TCMS) não apresenta consideráveis evidências de custo-benefício para a avaliação do parênquima mamário $^{(3)}$, entretanto, deve-se destacar seu importante papel no estadiamento e no seguimento de pacientes com câncer de mama, auxiliando na conduta terapêutica adotada, bem como no prognóstico do paciente.

No seguimento após tratamento adjuvante, não há consenso quanto à indicação formal de realização de TCMS na ausência de sintomas ou de indicações clínicas que justifiquem sua solicitação ${ }^{(4)}$, sendo mais sensível o método de tomografia por emissão de pósitrons ${ }^{(5)}$.

O objetivo do presente trabalho é demonstrar, por meio da TCMS, o aspecto das alterações extramamárias detectadas no estadiamento do câncer de mama e durante seguimento de pacientes submetidas a tratamento.

\section{APARÊNCIA PÓS-CIRÚRGICA DA PAREDE TORÁCICA}

A aparência pós-cirúrgica da parede torácica varia conforme a técnica cirúrgica executada.

Na mastectomia radical (Figura 1A) são removidos a mama, os músculos peitorais maior e menor e os linfonodos regionais ao longo da veia axilar até o ligamento costoclavicular.

$\mathrm{Na}$ mastectomia radical modificada, alternativa para o tratamento conservador, são realizadas mastectomia e remoção de linfonodos axilares, com preservação do músculo peitoral maior - procedimento de Patey (Figura 1B) -, ou com preservação de ambos os músculos peitorais maior e menor - procedimento de Auchincloss ${ }^{(6)}$.

Outros métodos mais conservadores/reconstrutores podem ser utilizados. A complicação cirúrgica mais comum é a ocorrência de seroma, podendo ocorrer ainda infecções, necrose, linfedema e contratura $\operatorname{axilar}^{(6)}$. 


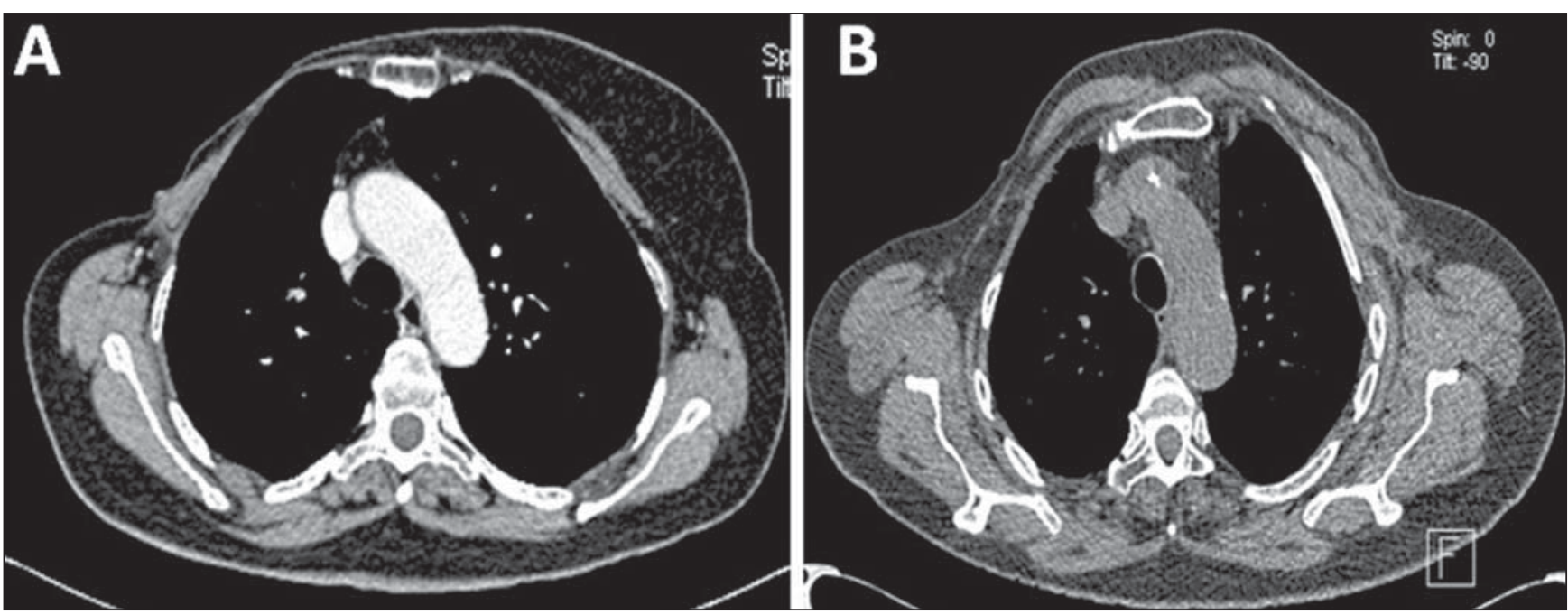

Figura 1. Aparência pós-cirúrgica da parede torácica à TCMS. A: Imagem axial de mastectomia total radical evidenciando a assimetria da parede torácica com ausência dos músculos peitoral maior e menor à direita. B: Imagem axial de mastectomia radical modificada por Patey, na qual é visualizada a ausência do músculo peitoral menor à direita.

\section{MANIFESTAÇÕES TORÁCICAS PÓS-RADIOTERAPIA}

A radioterapia é amplamente utilizada no pós-operatório de pacientes com câncer de mama, para reduzir o risco de recorrência locorregional e/ou volume tumoral em casos avançados ${ }^{(7)}$.

A radioterapia comumente ocasiona pneumonite por radiação, que ocorre cerca de 4 a 12 semanas após o término do tratamento e geralmente fica limitada ao campo da radiação. Inicialmente, manifesta-se como opacidades lineares ou como consolidações. Estas alterações podem desaparecer gradualmente ou podem acarretar sinais de alterações fibróticas evolutivas durante um período de seis meses a dois anos, com tendência a permanecerem estáveis após dois anos de evolução ${ }^{(6,8)}$ (Figura 2).

Há três campos de radiação que induzem pneumonite por radiação em pacientes com câncer de mama: tangencial, supraclavicular e mamário interno. O campo tangencial, utilizado para irradiar a parede torácica, resulta em pneumonite na periferia do pulmão anterolateralmente, com forma característica. No campo supraclavicular pode haver alterações nos ápices pulmonares, resultando em lesões semelhantes às vistas na tuberculose pulmonar. No campo mamário interno, via para irradiar os linfonodos homônimos, podem ser induzidas alterações na região paramediastinal. Quando são vistas áreas de opacidade em radiografias de seguimento de pacientes pós-radioterapia, o diagnóstico diferencial inclui pneumonite por radiação, recorrência local, disseminação de tumor linfangítico e pneumonite infecciosa ${ }^{(6)}$

\section{MANIFESTAÇÕES PÓS-QUIMIOTERAPIA}

Os principais agentes quimioterápicos utilizados no câncer de mama são a ciclofosfamida, o metotrexate, o 5-fluoracil e a doxorrubicina, e as principais manifestações pós-quimioterapia a serem consideradas incluem pneumonite, cardiotoxicidade e infecções.

A cardiotoxicidade geralmente é dosedependente decorrente da doxorrubicina, inicialmente assintomática, e evolui para quadros de arritmias transitórias até cardiomiopatia fatal por disfunção permanente do ventrículo esquerdo. Manifesta-se à TCMS como cardiomegalia e/ou derrame pericár$\operatorname{dico}^{(\mathbf{9})}$ (Figura 3).

Doenças pulmonares intersticiais compõem um amplo e heterogêneo grupo de doenças pulmonares fibróticas, incluindo pneumonite intersticial. A maioria dos casos de pneumonite intersticial tem causa desconhecida. A toxicidade pulmonar induzida por fármacos aponta para uma possível etiologia subdiagnosticada de doenças pulmonares intersticiais. Tem-se demonstrado que os tratamentos atualmente preconizados para câncer de mama, incluindo tamoxifeno e taxanos, aumentam o risco de pneumonite intersticial, especialmente quando combinados a radioterapia adjuvante. As alterações pulmonares mais frequentes decorrentes de toxicidade por drogas quimioterápicas são padrão intersticial, opacidade "em vidro fosco" e consolidações (Figura 4), e estas, ocasionalmente, podem ter uma aparência que simula um nódulo ou uma massa ${ }^{(10)}$.

\section{RECORRÊNCIA LOCORREGIONAI}

Recorrência local é definida como ressurgimento tumoral no sítio cirúrgico, que ocorre em 34-84\% dos casos, e a recorrência regional, pelo aparecimento de metástases em linfonodos que realizam a drenagem linfática da mama, incluindo linfonodos supraclaviculares, axilares e a cadeia da mamária interna ${ }^{(\mathbf{1 1})}$.

A detecção do câncer de mama recorrente, pela mamografia, é desafiadora por causa das alterações arquiteturais, fibrose e cicatrizes do parênquima mamário, secundárias a cirurgia e radioterapia, as quais dificultam a interpretação do exame ${ }^{(\mathbf{1 2})}$.

Geralmente, pacientes com envolvimento de quatro ou mais linfonodos axilares têm risco significativo de recorrência, demonstrada como linfonodos múltiplos e aumentados de diâmetro à TCMS. Frequentemente, as metástases para linfonodos da cadeia mamária interna (Figura 5) e mediastinais são clinicamente ocultas por 


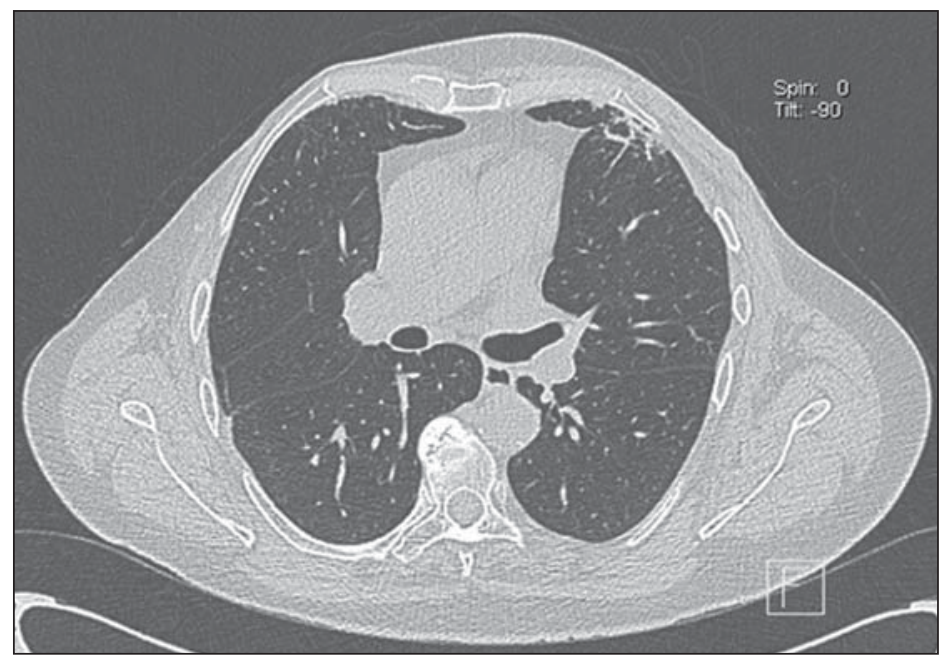

Figura 2. Manifestação torácica pós-radioterapia. Imagem axial de TCMS demonstrando lesão actínica no pulmão esquerdo em paciente submetida a radioterapia por câncer de mama.

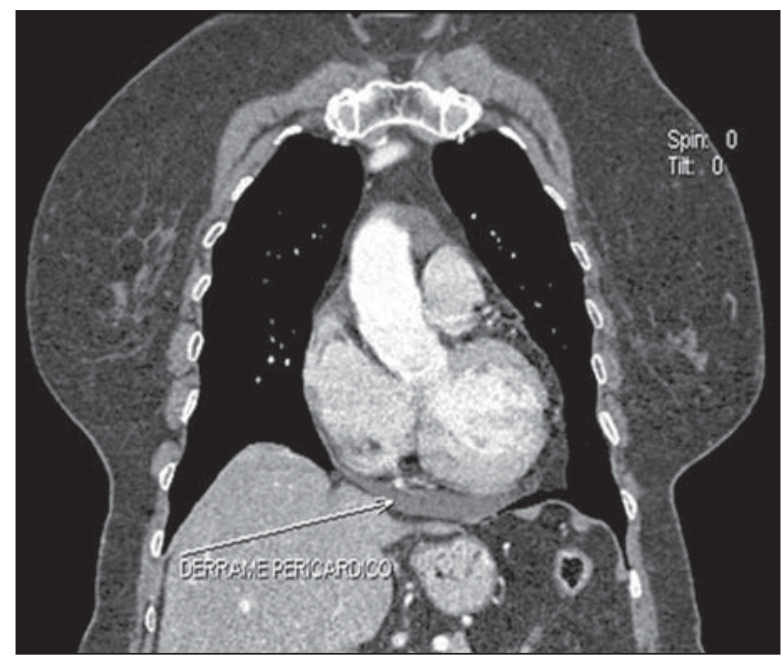

Figura 3. Manifestação pós-quimioterapia. Imagem coronal de TCMS evidenciando derrame pericárdico em paciente em tratamento quimioterápico para câncer de mama.

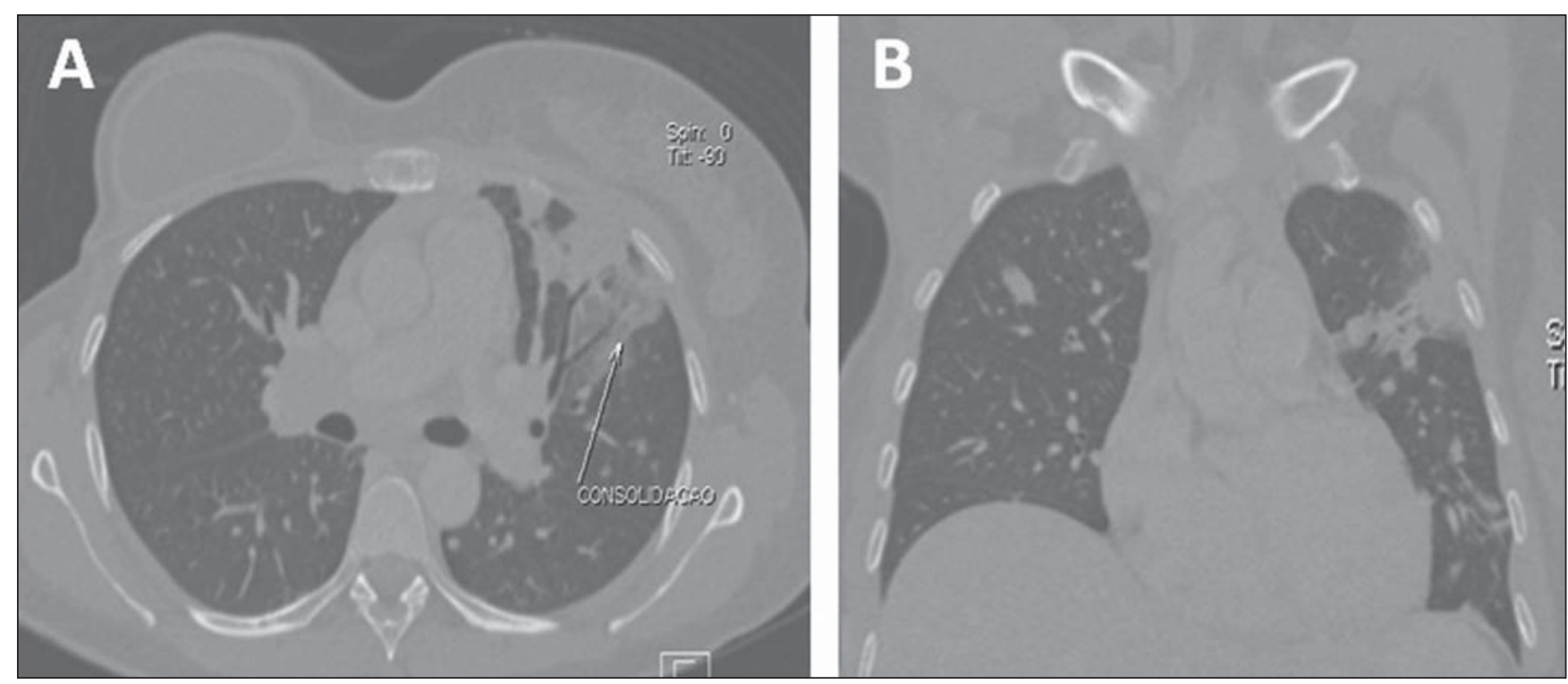

Figura 4. Manifestação pós-quimioterapia. Imagens axial (A) e coronal (B) de TCMS evidenciando consolidação pulmonar decorrente de quimioterapia em paciente com câncer de mama.

serem pequenas, produzindo achados equívocos ou negativos à TCMS ${ }^{(13)}$.

Os sinais tomográficos sugestivos de recorrência local incluem espessamento cutâneo focal maior que $1 \mathrm{~cm}$, presença de lesões nodulares no tecido subcutâneo ou na musculatura da parede torácica, e irregularidade dos contornos e heterogeneidade da musculatura peitoral ${ }^{(11)}$.

Os linfonodos normais da cadeia da mamária interna têm menos que $5 \mathrm{~mm}$ de diâmetro e as metástases para esta cadeia não são facilmente detectadas pelo exame físico, mamografia ou ultrassonografia, porque se situam recobertas por estruturas ósseas, cartilaginosas ou ósseas da parede torácica. Linfonodos normais na cadeia mamária interna não são identificados de rotina pela tomografia computadorizada. Portanto, linfonodos na cadeia mamária interna detectáveis e com diâmetro maior que $6 \mathrm{~mm}$ em paciente com câncer de mama sugerem linfonodopatia maligna ${ }^{(\mathbf{1 4})}$.

A recorrência na cadeia mamária interna é rara, ocorrendo com bastante variação em 8-37\% dos casos, entretanto, está concomitantemente presente em $44 \%$ dos casos em que há envolvimento axilar ${ }^{(11)}$.

\section{COMPROMETIMENTO LINFONODAL}

As metástases linfonodais (Figuras 6 e 7) são relativamente frequentes no câncer de mama e o estadiamento adequado do tumor é importante para otimizar a propedêutica a ser adotada ${ }^{(15)}$

Apesar de ser um método comprovadamente capaz de reduzir a morbidade, a biópsia do linfonodo sentinela é um procedimento invasivo ${ }^{(\mathbf{1 6})} \mathrm{e}$, portanto, a obtenção de imagens e nodos linfáticos pela TCMS com uso de contraste parece ser um método 

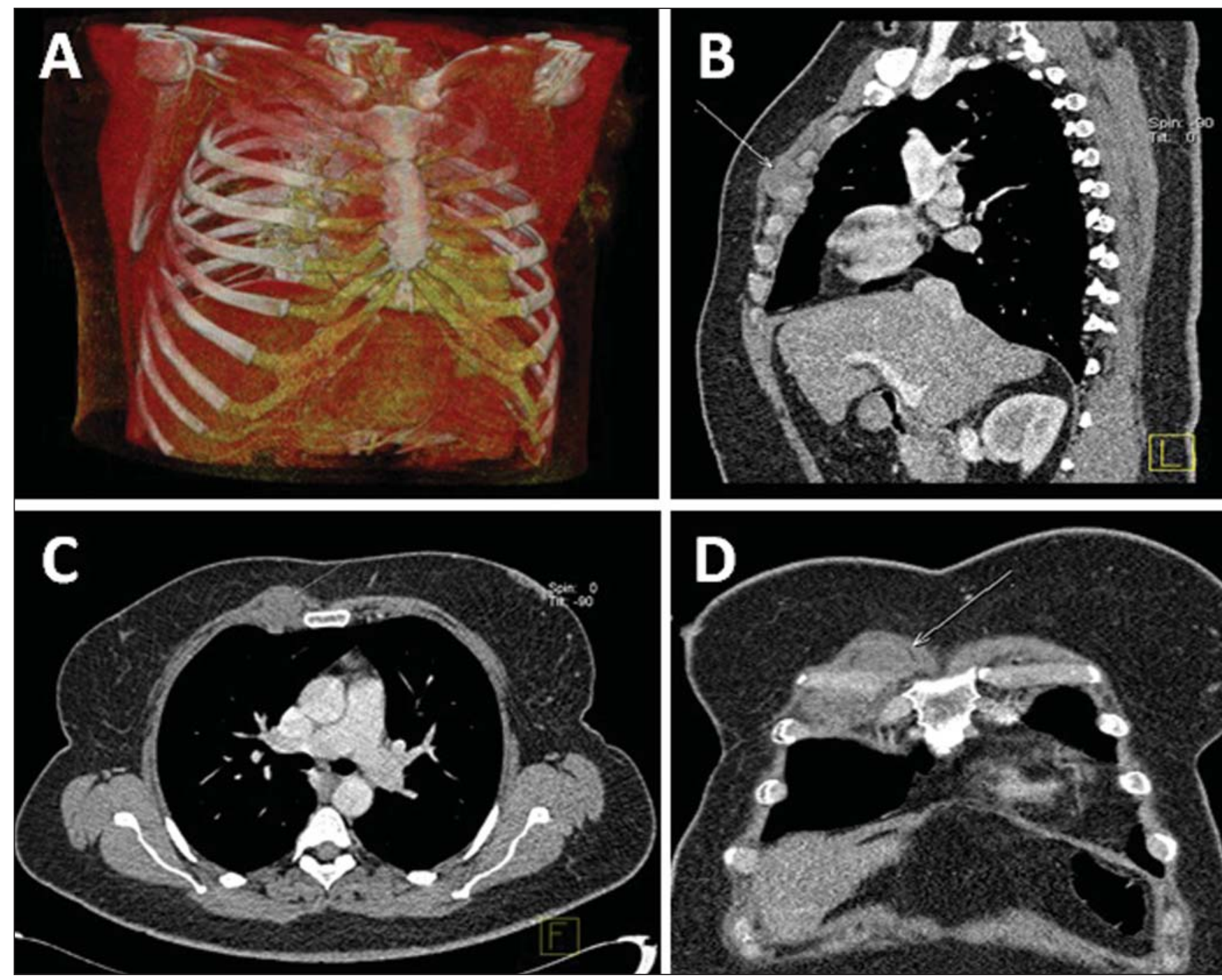

Figura 5. Linfonodomegalia na cadeia mamária interna direita. Recorrência regional após seis meses de tratamento cirúrgico mamário conservador. TCMS do tórax, técnica de renderização de volume (A), imagens sagital (B), axial (C) e coronal (D).
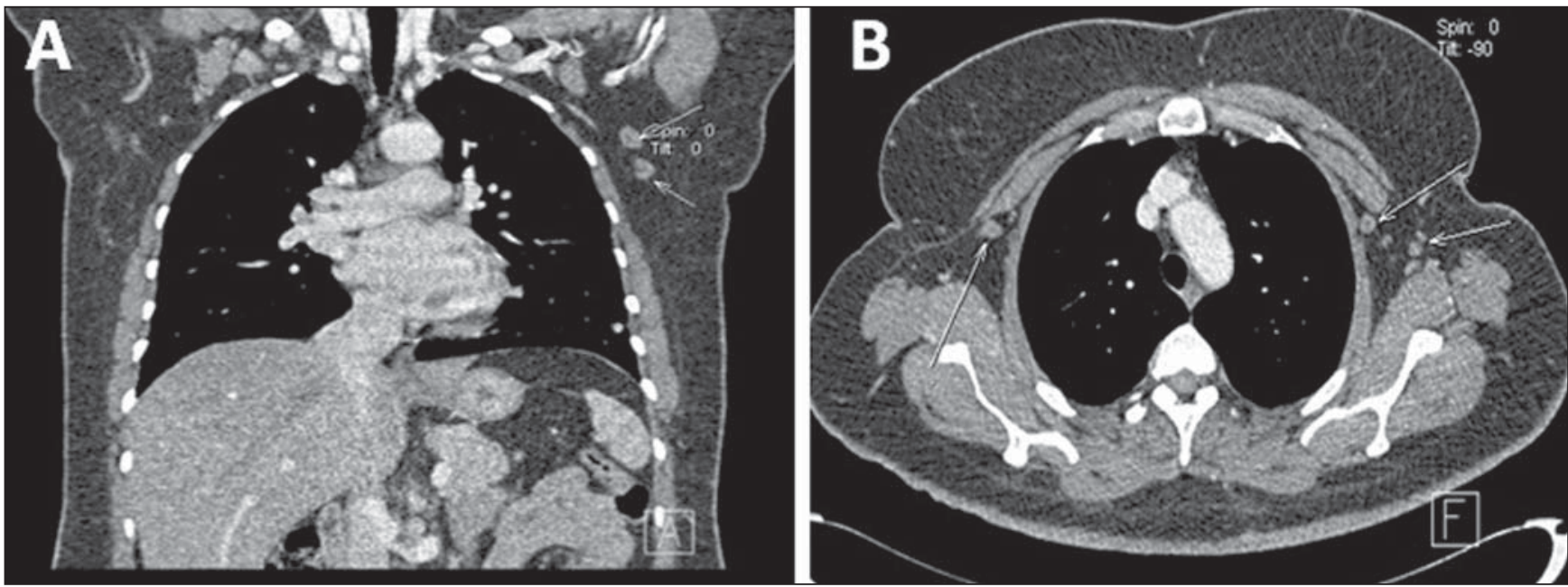

Figura 6. Comprometimento linfonodal. Imagens coronal (A) e axial (B) de TCMS evidenciando linfonodos axilares bilateralmente, alguns deles com dimensões aumentadas. 


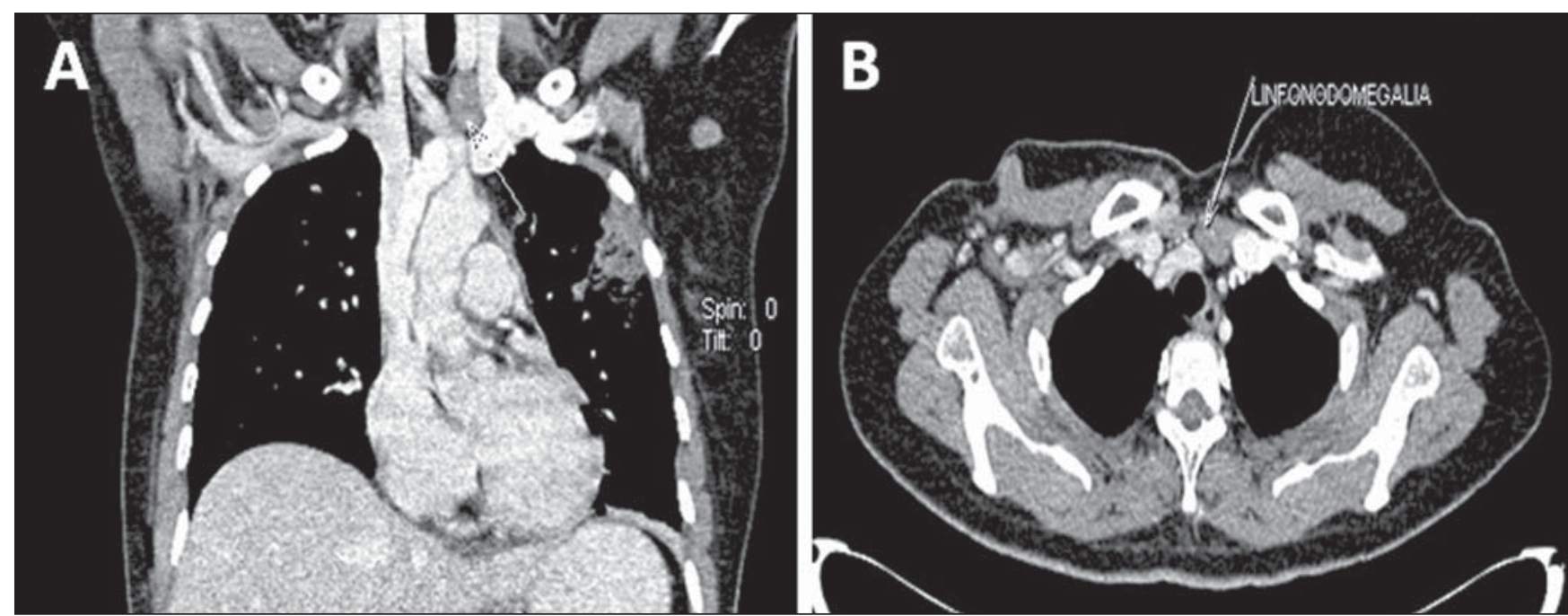

Figura 7. Comprometimento linfonodal. Imagens coronal (A) e axial (B) de TCMS evidenciando linfonodomegalia supraclavicular e axilar à esquerda.

mais conveniente, mesmo apesar de indireto $^{(17)}$

A tomografia é a modalidade de imagem de escolha para a detecção do comprometimento linfonodal, muitas vezes definindo o estadiamento do paciente pela classificação TNM, apesar de não possuir valor na avaliação do tamanho do tumor. Além disso, seu desempenho quanto à detecção e quantificação do câncer é significativamente reduzido quando as lesões têm

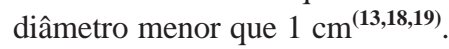

\section{METÁSTASE PLEURAL E PULMONAR}

A pleura é alvo frequente de metástase em pacientes com câncer de mama (Figura 8), sendo o derrame pleural ipsilateral ao tumor primário a manifestação mais comum desta metástase, por provável disseminação linfática. Metástase de câncer de mama é uma das maiores três causas para o derrame maligno ${ }^{(20)}$.

Nodularidade, espessamentos irregulares e placas pleurais são achados menos comuns em metástase pleural e raramente ocorrem sem derrame pleural associado ${ }^{(6)}$.

Achados comuns em metástase pulmonar de câncer de mama são os múltiplos nódulos (Figura 9) que ocorrem por disseminação hematogênica do tumor. Geralmente, as lesões metastáticas apresentam forma esférica ou ovoide, tamanho variável, margem bem delimitada e estão localizadas principalmente na periferia do pul-

Figura 8. Metástase pleural. Imagem axial de TCMS mostrando metástase pleural em paciente com câncer de mama. Notar metástases hepáticas calcificadas (paciente submetida a quimioterapia prévia) e ascite.

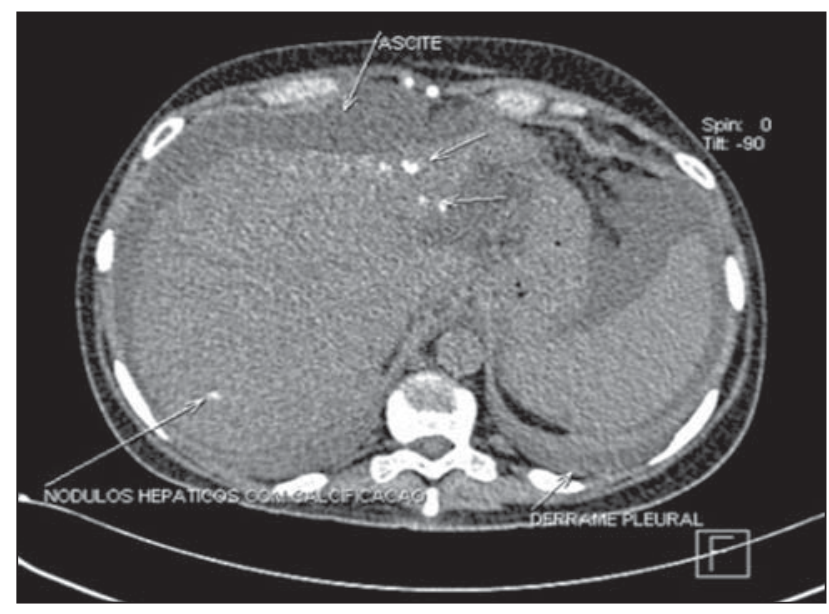

mão, podendo ser encontradas como nódulos calcificados ${ }^{(\mathbf{6 , 2 0 , 2 1})}$.

A detecção de nódulo pulmonar solitário em pacientes em tratamento prévio para câncer de mama não representa necessariamente doença metastática. Em grande parcela desses pacientes, o nódulo pulmonar solitário é decorrente de um carcinoma pulmonar primário. Porém, em pacientes com malignidade extratorácica, a chance de metástase representa $25 \%{ }^{(\mathbf{6 , 2 1})}$.

Outras manifestações de metástase pulmonar são a linfangite carcinomatosa e os nódulos centrolobulares, ambos decorrentes de disseminação endobrônquica ${ }^{(6)}$.

\section{METÁSTASE ÓSSEA}

O segundo tipo mais frequente de metástase a distância por câncer de mama é a metástase óssea, que causa elevada morbidade devido a dor, comprometimento da mobilidade, hipercalcemia, fratura patológica, compressão do saco dural, da medula espinhal ou de raízes nervosas e infiltração da medula óssea. O câncer de mama é a causa mais comum de compressão medular em mulheres ${ }^{(\mathbf{6})}$.

Várias modalidades de exames de imagem estão disponíveis para avaliação de metástases ósseas em pacientes com câncer de mama. Sugere-se a utilização da cintilografia óssea como primeira técnica de imagem em pacientes assintomáticos, pois o exame é bastante sensível na detecção de metástases ósseas e permite excelente avaliação óssea ${ }^{(22)}$.

Em radiologia, a maioria das metástases ósseas é múltipla e pode ser lesões osteolíticas, osteoblásticas ou uma combina- 


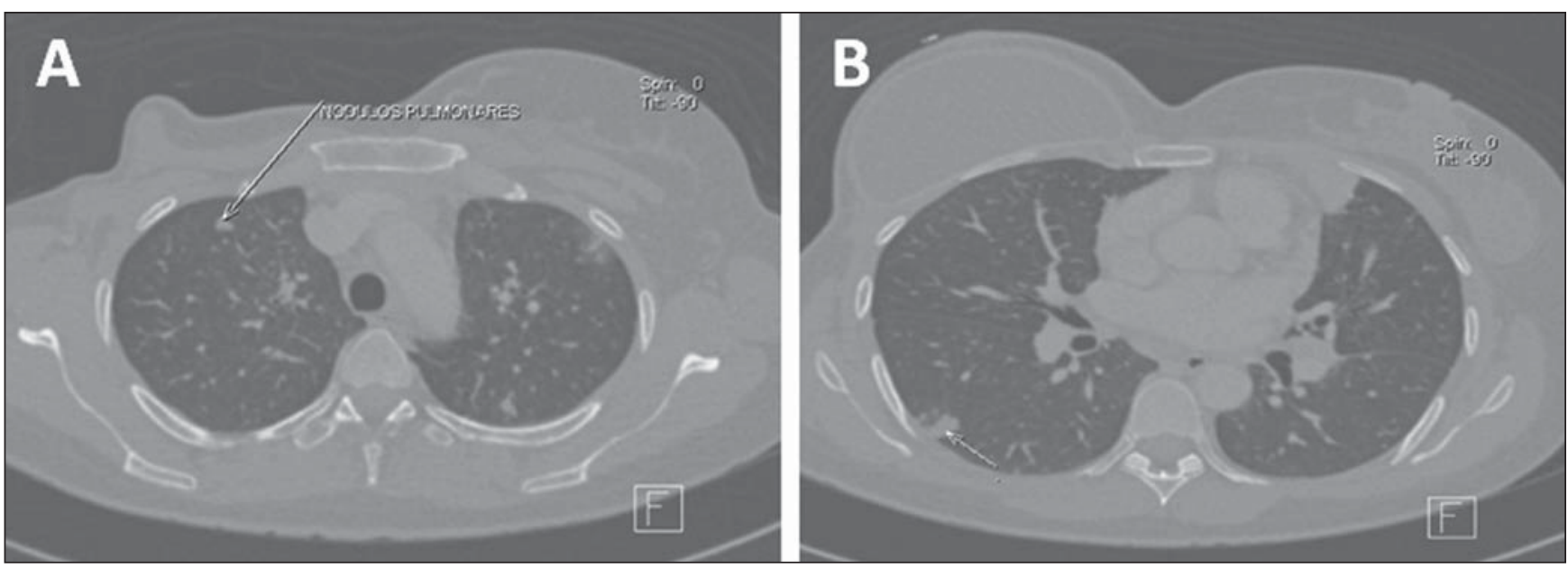

Figura 9. Metástase pulmonar. Imagens axiais de TCMS evidenciando nódulos pulmonares metastáticos em paciente submetida a mastectomia total à direita por câncer de mama.

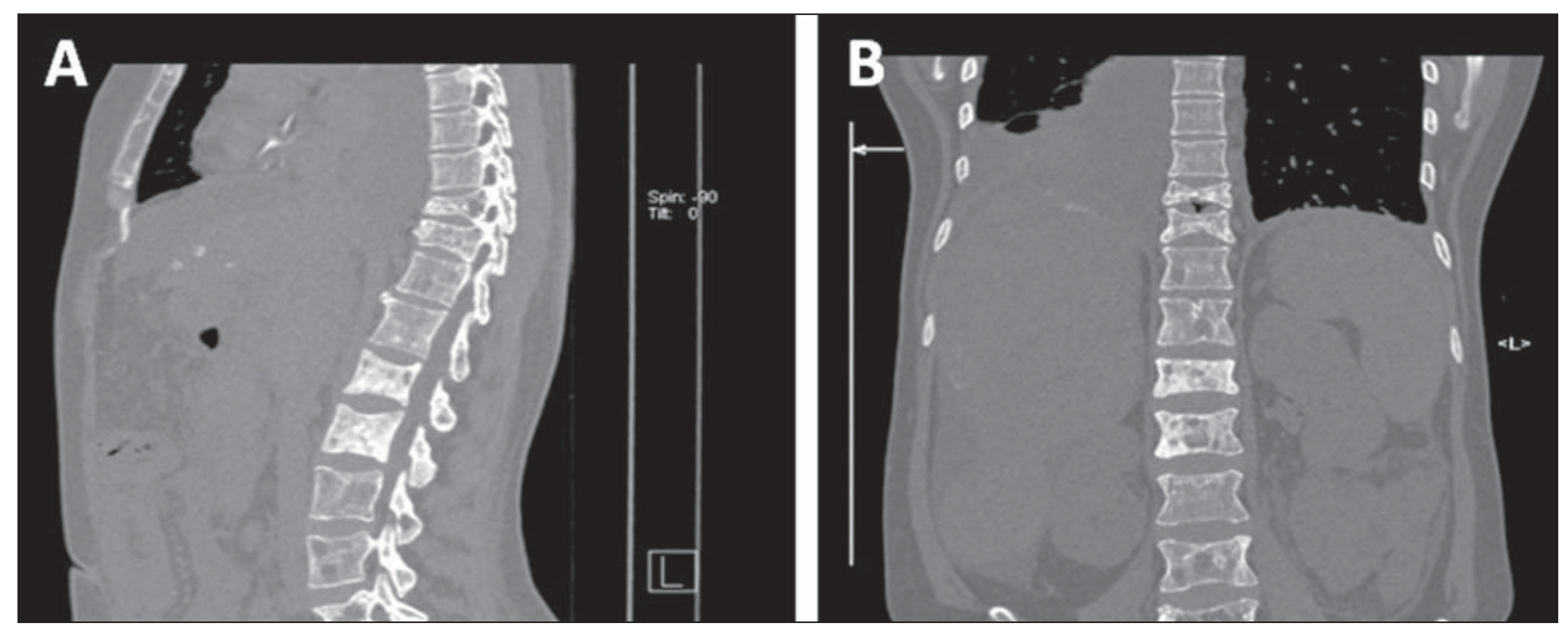

Figura 10. Metástases ósseas. Imagens sagital (A) e coronal (B) de TCMS evidenciando metástases ósseas do tipo mista (osteolítica e osteoblática) na coluna dorsal e lombar em paciente com câncer de mama e dor dorsolombar.

ção de ambas ${ }^{(6,23,24)}$ (Figura 10). É importante ressaltar que, após radioterapia, quimioterapia ou tratamento hormonal, metástases ósseas inicialmente osteolíticas podem se tornar osteoescleróticas.

\section{METÁSTASE HEPÁTICA}

Aproximadamente metade das mulheres com câncer de mama metastático apresenta metástase hepática durante o curso da doença. Radiograficamente, as metástases hepáticas podem ter diversas formas: apresentam-se como lesões "em alvo" à ultrassonografia e lesões hipoatenuantes na fase portal à tomografia computadorizada, devido à hipovascularização delas ${ }^{(25,26)}(\mathrm{Fi}$ - gura 11). Geralmente são lesões nodulares não calcificadas, podendo apresentar calcificações após tratamento quimioterápico (Figura 8).

Estudos têm demonstrado que metástases hepáticas podem ser identificadas como hipervascularizadas na fase arterial do contraste. As lesões hipervascularizadas são menos comuns e podem ser isoatenuantes durante a fase portal ${ }^{(26,27)}$.

O padrão típico de realce de metástase de câncer de mama é um discreto realce periférico nas fases arterial e mais intensamente na fase portal. Outro padrão típico de realce pós-contraste de metástase de câncer de mama são lesões com mínimo realce periférico ou sem realce periférico pós-contraste venoso na fase arterial e sem realce na fase portal, tornando-se evidentes nesta fase por se apresentarem hipoatenuantes em relação ao parênquima hepático.

\section{METÁSTASE CEREBRAL}

O câncer de mama é responsável por aproximadamente $10-15 \%$ dos casos de metástases cerebrais (Figura 12), sendo, em 70-80\% dos casos, lesões múltiplas, que muitas vezes são diagnosticadas após alterações nos demais sistemas orgânicos. A localização de maior frequência é a junção entre as substâncias branca e cinzenta e as fronteiras vasculares, seguidas das estruturas parenquimatosas profundas e posterior- 

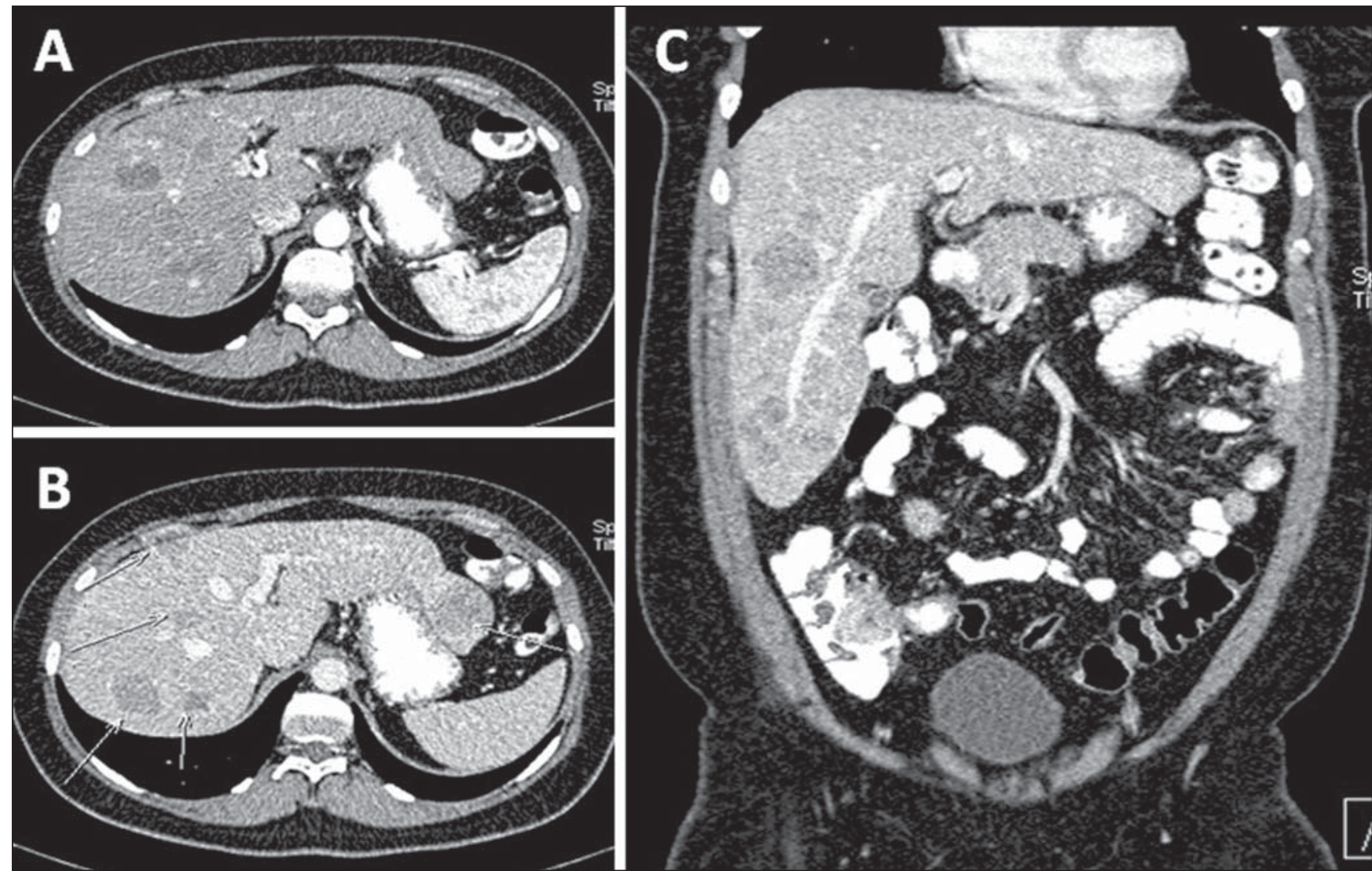

Figura 11. Metástases hepáticas. Imagens axiais nas fases arterial (A) e portal (B) e coronal na fase portal (C) evidenciando metástases hepáticas hipovascularizadas em paciente com câncer de mama.
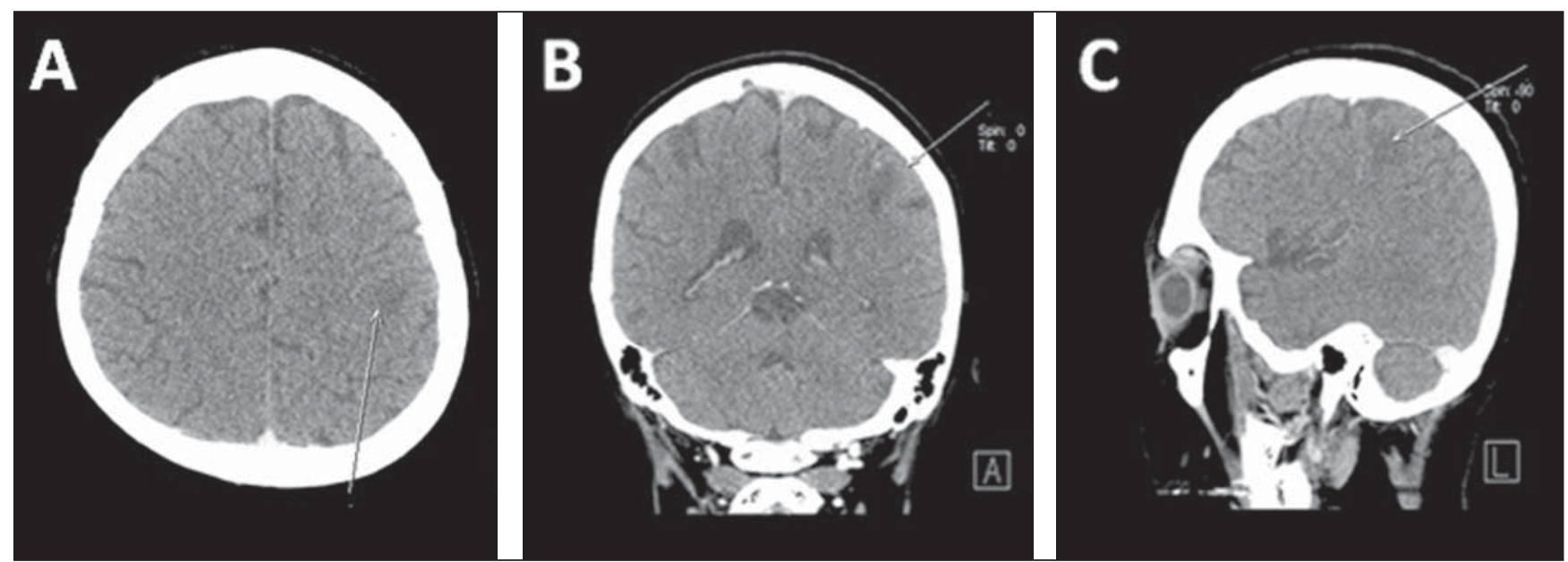

Figura 12. Metástase cerebral. Imagens axial (A), coronal (B) e sagital (C) de TCMS evidenciando metástase cerebral em paciente com câncer de mama.

mente para o tronco cerebral, sendo as regiões supratentoriais mais frequentes que as infratentoriais. Metástases cerebrais também podem ocorrer para as leptomeninges $(2-5 \%)$ e dura-máter ${ }^{(28)}$.

A maioria das metástases cerebrais manifesta-se como lesões isoatenuantes ou discretamente hiperatenuantes, podendo ainda ser hipoatenuantes, com edema perilesional e com realce pós-contraste venoso. Hemorragias, alterações císticas e necroses são comuns em todos os tipos de tumores metastáticos. Todavia, não há achados patognomônicos que distinguem metástases cerebrais de tumores malignos primários cerebrais ou condições não neoplásicas ${ }^{(29)}$.

\section{CONCLUSÃO}

Apesar de a TCMS não ser o método imaginológico de escolha para a avaliação do parênquima mamário, sua utilização permite a identificação de dados importantes em pacientes sintomáticos, naqueles que apresentam alterações radiográficas, 
em pacientes nos quais foram constatadas lesões à ultrassonografia abdominal e, também, no acompanhamento da resposta ao tratamento quimioterápico. O diagnóstico precoce permanece como o melhor método para aumentar as chances de cura no câncer de mama.

\section{REFERÊNCIAS}

1. Brasil. Ministério da Saúde. Instituto Nacional de Câncer. Estimativa 2010: incidência de câncer no Brasil. Rio de Janeiro, RJ: INCA; 2009.

2. Lee CH, Dershaw DD, Kopans D, et al. Breast cancer screening with imaging: recommendations from the Society of Breast Imaging and the ACR on the use of mammography, breast MRI, breast ultrasound, and other technologies for the detection of clinically occult breast cancer. J Am Coll Radiol. 2010;7:18-27.

3. Chala LF, Barros N. Avaliação das mamas com métodos de imagem. Radiol Bras. 2007;40(1):ivvi.

4. Brasil. Instituto Nacional de Câncer. Controle do câncer de mama. Documento de consenso. Rio de Janeiro, RJ: INCA; 2004.

5. Soares Junior J, Fonseca RP, Cerci JJ, et al. Lista de Recomendações do Exame PET/CT com ${ }^{18} \mathrm{~F}$ FDG em Oncologia. Consenso entre a Sociedade Brasileira de Cancerologia e a Sociedade Brasileira de Biologia, Medicina Nuclear e Imagem Molecular. Radiol Bras. 2010;43:255-9.

6. Jung JI, Kim HH, Park SH, et al. Thoracic manifestations of breast cancer and its therapy. Radiographics. 2004;24:1269-85.

7. Formenti SC, Demaria S. Local control by radiotherapy: is that all there is? Breast Cancer Res. 2008; 10:215.

8. Krengli M, Sacco M, Loi G, et al. Pulmonary changes after radiotherapy for conservative treat- ment of breast cancer: a prospective study. Int $\mathrm{J}$ Radiat Oncol Biol Phys. 2008;70:1460-7.

9. Bird BR, Swain SM. Cardiac toxicity in breast cancer survivors: review of potential cardiac problems. Clin Cancer Res. 2008;14:14-24.

10. Christensen S, Pedersen L, Grijota M, et al. Incidence of interstitial pneumonitis among breast cancer patients: a 10-year Danish populationbased cohort study. Br J Cancer. 2008;98:1870 5.

11. Chen L, Gu Y, Leaw S, et al. Internal mammary lymph node recurrence: rare but characteristic metastasis site in breast cancer. BMC Cancer. 2010;10:479.

12. Bénard F, Turcotte E. Imaging in breast cancer: single-photon computed tomography and positron-emission tomography. Breast Cancer Res. 2005; 7:153-62.

13. Yang SK, Cho N, Moon WK. The role of PET/CT for evaluating breast cancer. Korean J Radiol. 2007;8:429-37.

14. Cody HS 3rd, Urban JA. Internal mammary node status: a major prognosticator in axillary nodenegative breast cancer. Ann Surg Oncol. 1995;2: 32-7.

15. Jager JJ, Keymeulen K, Beets-Tan RG, et al. FDGPET-CT for staging of high-risk breast cancer patients reduces the number of further examinations: a pilot study. Acta Oncol. 2010;49:185-91.

16. Chae BJ, Bae JS, Kang BJ, et al. Positron emission tomography-computed tomography in the detection of axillary lymph node metastasis in patients with early stage breast cancer. Jpn J Clin Oncol. 2009;39:284-9.

17. Takahashi M, Sasa M, Hirose C, et al. Clinical efficacy and problems with CT lymphography in identifying the sentinel node in breast cancer. World J Surg Oncol. 2008;6:57.

18. Yamashita K, Shimizu K. Evaluation of sentinel lymph node metastasis alone guided by three-dimensional computed tomographic lymphography in video-assisted breast surgery. Surg Endosc 2009;23:633-40.

19. Bowen SL, Wu Y, Chaudhari AJ, et al. Initial characterization of a dedicated breast PET/CT scanner during human imaging. J Nucl Med. 2009;50: 1401-8

20. Avdalovic M, Chan A. Thoracic manifestations of common nonpulmonary malignancies of women Clin Chest Med. 2004;25:379-90.

21. Seo JB, Im J, Goo JM, et al. Atypical pulmonary metastases: spectrum of radiologic findings. Radiographics. 2001;21:403-17.

22. Costelloe CM, Rohren EM, Madewell JE, et al Imaging bone metastases in breast cancer: techniques and recommendations for diagnosis. Lancet Oncol. 2009;10:606-14.

23. Koizumi M, Yoshimoto M, Kasumi F, et al. Comparison between solitary and multiple skeletal metastatic lesions of breast cancer patients. Ann Oncol. 2003;14:1234-40.

24. Mundy GR. Metastasis to bone: causes, consequences and therapeutic opportunities. Nat Rev Cancer. 2002;2:584-93.

25. Diamond JR, Finlayson CA, Borges VF. Hepatic complications of breast cancer. Lancet Oncol. 2009; 10:615-21.

26. Roach H, Whipp E, Virjee J, et al. A pictorial review of the varied appearance of atypical liver metastasis from carcinoma of the breast. Br J Radiol. 2005;78:1098-103.

27. Blake MA, McDermott S, Rosen MP, et al. Expert panel on gastrointestinal imaging. ACR Appropriateness Criteria ${ }^{\circledR}$ suspected liver metastases. [online publication]. Reston, VA: American College of Radiology; 2011.

28. Wadasadawala T, Gupta S, Bagul V, et al. Brain metastases from breast cancer: management approach. J Cancer Res Ther. 2007;3:157-65.

29. Soffietti R, Rudā R, Mutani R. Management of brain metastases. J Neurol. 2002;249:1357-69. 